\title{
Cloud-Based Fault Tolerant Control for a DC Motor System
}

\author{
Xiao He, ${ }^{1}$ Yamei Ju, ${ }^{2,3}$ Yang Liu, ${ }^{4}$ and Bangcheng Zhang ${ }^{2,5}$ \\ ${ }^{1}$ Department of Automation, TNList, Tsinghua University, Beijing 100084, China \\ ${ }^{2}$ School of Mechatronic Engineering, Changchun University of Technology, Jilin 130012, China \\ ${ }^{3}$ Guangdong Provincial Key Laboratory of Petrochemical Equipment Fault Diagnosis, \\ Guangdong University of Petrochemical Technology, Maoming 525000, China \\ ${ }^{4}$ College of Electrical Engineering and Automation, Shandong University of Science and Technology, Qingdao 266590, China \\ ${ }^{5}$ Automotive Engineering Research Institute, Changchun University of Technology, Jilin 130012, China
}

Correspondence should be addressed to Bangcheng Zhang; zhangbangcheng@mail.ccut.edu.cn

Received 28 August 2016; Accepted 23 October 2016; Published 16 March 2017

Academic Editor: Francisco Gordillo

Copyright (C) 2017 Xiao He et al. This is an open access article distributed under the Creative Commons Attribution License, which permits unrestricted use, distribution, and reproduction in any medium, provided the original work is properly cited.

\begin{abstract}
The fault tolerant control problem for a DC motor system is investigated in a cloud environment. Packet dropout phenomenon introduced by the limited-capacity communication channel is considered. Actuator faults are taken into consideration and fault diagnosis and fault tolerant control methods towards actuator faults are proposed to enhance the reliability of the whole cloud-based DC motor system. The fault diagnosis unit is then established with purpose of obtaining fault information. When the actuator fault is detected by comparing the residual signal with a predefined threshold, a residual matching approach is utilized to locate the fault. The fault can be further estimated by a least-squares filter. Based on the fault estimation, a fault tolerant controller is designed to guarantee the stability as well as the control performance of the DC motor system. Simulation result on a DC motor system shows the efficiency of the fault tolerant control method proposed in this paper.
\end{abstract}

\section{Introduction}

DC motors have been widely used in various fields for more than one century and they have been playing more and more important roles in modern manufacturing. In traditional DC motor control technique, control signals are transmitted from the controller node to the motor by point-to-point wiring and a specialized controller is designed to control the DC motor with prescribed performance. In the past decades, network technology has developed dramatically and various types of network have been utilized to control systems, stimulating the wide investigation of Networked Control Systems (NCSs) [1-3]. Figure 1 shows a typical structure of NCSs where the system, controller, sensor, and actuator are connected through a network and information is exchanged using a limited-capacity communication channel. The use of a communication network offers advantages in terms of reliability, enhanced resource utilization, reduced wiring, and reconfigurability.
Very recently, the idea of cloud-based control systems has been proposed and it is now being formulated along with the developments of the Internet of Things (IoT) and CyberPhysical Systems (CPSs) $[4,5]$. In the cloud-based control framework, the hardware of the controller can be removed and the controller can be customized from the cloud. As is shown in Figure 2, this type of control strategy combines the network resources and computational resources and will greatly reduce the costs of different control tasks. In the cloud-based control systems, there is not a specialized controller for a specific controlled system, and the controller is realized randomly by computational nodes shared in the cloud. Each node in the cloud can be accessed by the controlled system using network and one node will contribute computational resource to perform control/filter tasks once it is chosen according to a certain rule. The cloud-based control system inherits the advantages of the NCSs, such as easy installation, low cost of maintenance, and flexible architecture. Furthermore, cloud-based control system implements 


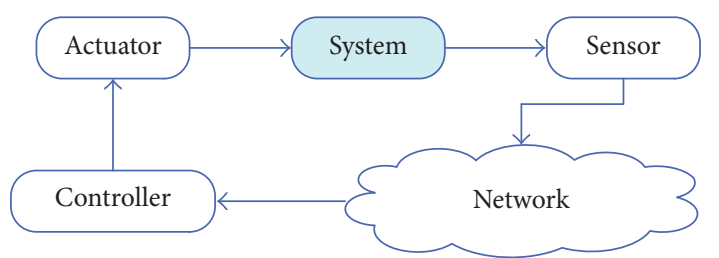

FIGURE 1: A typical structure of Networked Control System.

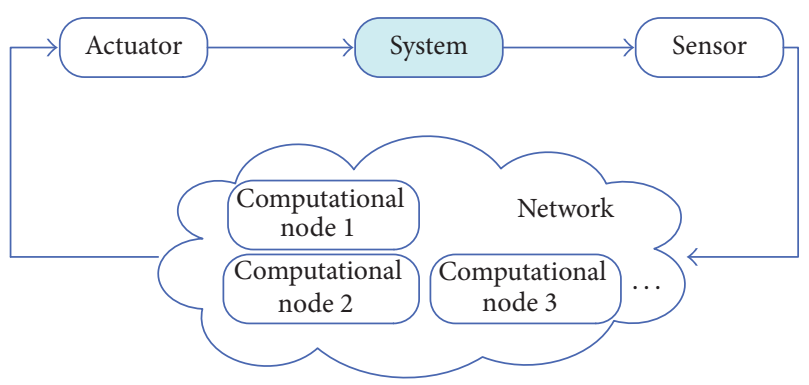

FIgURE 2: A typical structure of cloud-based control system.

the control algorithm by using the computational node in shared cloud. This control structure can further reduce the cost of specialized computational resources and benefits from the flexibility of remote monitoring and adjustment of system states and parameters over the communication channel. Although cloud-based control systems have more attractive advantages than NCSs, they are less reliable when being applied in real engineering practice due to their uncertain control/filter resource suppliers and uncertain communication links. In cloud-based control systems, data can be transmitted and exchanged through a shared network and the introduction of the communication network may induce more unexpected phenomena, such as time delays, quantization, and packet dropouts. These phenomena inevitably degrade the reliability of the system [6-12]. To collect and process the data efficiently, the mass data in control system will make the network burden and computational burden heavy.

In order to improve the safety of the control system, fault detection and isolation (FDI) and fault tolerant control (FTC) techniques have been proved as effective techniques [13]. FDI technique can detect the occurrence of a fault and determine the location of the fault, as well as estimating its amplitude. As a key step of active FTC, an FDI unit provides exact fault information and helps the fault accommodation unit to compensate the occurred fault by adjusting the controller parameter or controller structure. Active FTC has superiority over passive FTC framework in the control performance in the faulty case and the ability to handle unknown faults [14-21]. Active FTC problem has been investigated and many applications have been reported towards industrial or commercial processes in the past three decades [22-27]. In [28], the datadriven output-feedback fault tolerant control (FTC) problem has been studied for unknown dynamic systems with faults changing system dynamics. In this paper, an output-feedback approximate dynamic programming method is developed to solve the optimal control problem under the condition of the unknown linear time-invariant discrete-time plant. In [29], the FTC problem has been studied for a class of Networked Control Systems with random time delays and actuator failures. Reference [30] has dealt with the problem of the FTC design of continuous-time linear systems by the dynamic output controllers in the active fault tolerant structure with virtual actuators. Comparing the fruitful results on the frontiers of FTC theory and application, little work has been done towards the active FTC problem in a cloud-based environment, which motivates our present study.

We consider a DC motor as the controlled system and the active FTC problem for a DC motor system is investigated in a cloud environment. The system structure is shown in Figure 3. The packet dropouts of the measurement signal are considered. Actuator faults are taken into consideration and fault diagnosis and fault tolerant control methods towards actuator faults are proposed to enhance the reliability of the whole cloud-based DC motor system. A redundancy filter design technique is proposed, where each filter is implemented by a certain computational node in the cloud and is designed to estimate the augmented signal composed of the system state and a specific fault signal. A residual matching approach is put forward that can be used for the fault detection and fault isolation. We consider the filter with least residual as the one corresponding to the right fault, and the fault can be estimated by resorting to the output of the filter with the least residual. Then the fault compensator controller can accommodate the fault and reconfigure the control law to maintain the control performance of the DC motor. The main difference between the work in this paper and the existing work in the literature is that we do not fix a controller to perform the FTC and the computational resources in the cloud compete for being the real fault tolerant controller. The fault diagnosis unit is established with purpose of obtaining fault information. When the actuator fault is determined, a fault tolerant controller is designed to guarantee the stability as well as the control performance of the DC motor system. Simulation results on the cloud-based DC motor system are employed to illustrate the efficiency of the proposed method in this paper.

The main contributions can be summarized as follows: (1) a novel system structure and formulation is proposed for the active FTC problem in a cloud environment, where the fault diagnosis is implemented by the computational resources in the cloud; (2) a residual matching approach is carried out to implement the fault isolation and the fault estimation can be accomplished by least-squares filter recursively; (3) the problem is solved with packet dropout and actuator fault for the cloud-based DC motor system and simulation result shows the effectiveness of the proposed technique.

Notations. The notations used throughout the paper are fairly standard except where otherwise stated. $\mathbb{R}^{n}$ is the $n$ dimensional Euclidean space; $\mathbb{R}^{n \times m}$ is the set of all $n \times m$ real matrices. $P>0$ means that $P$ is real symmetric and positive definite. $A^{T}$ denotes the transpose of a matrix $A . E\{x\}$ stands for the expectation of a random variable $x$. 


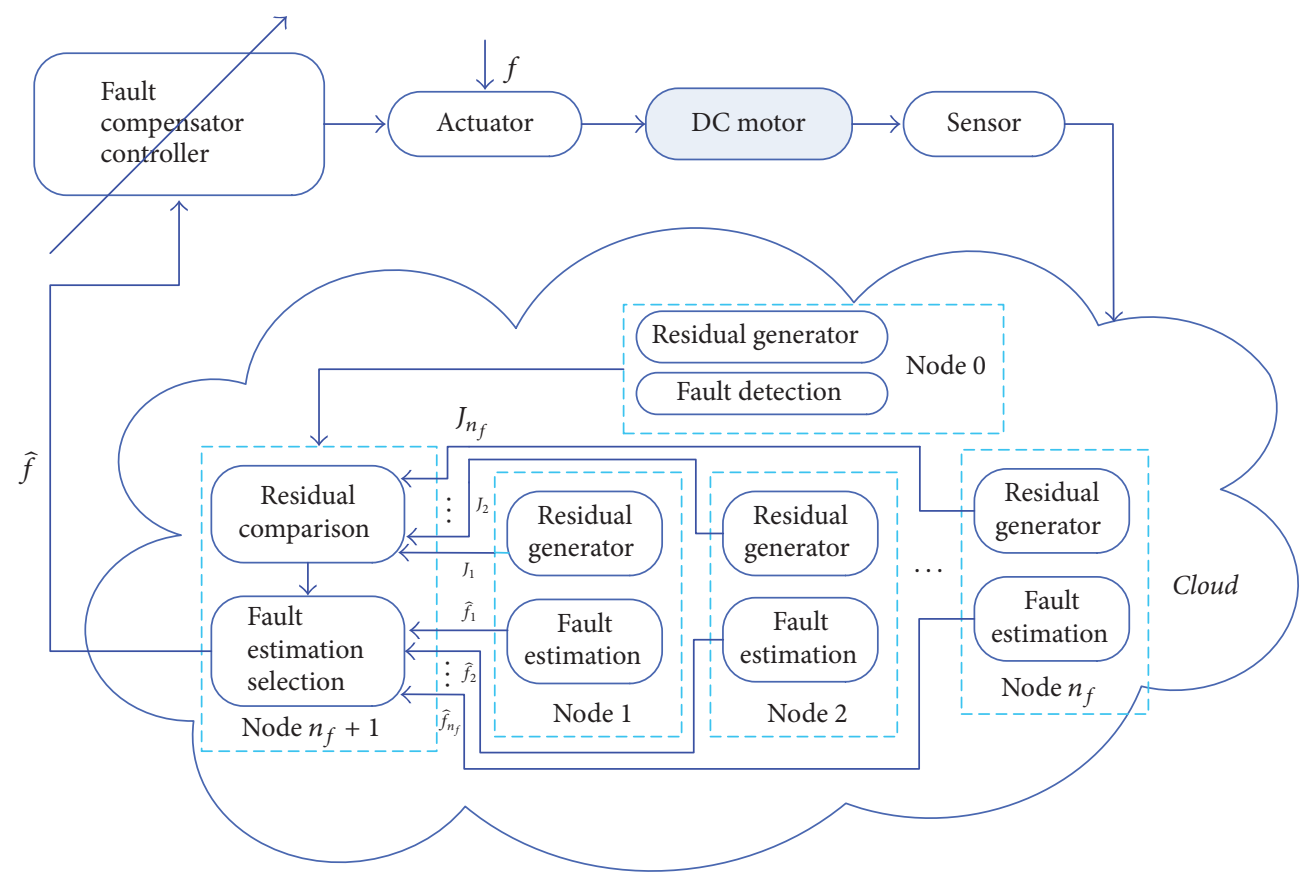

FIGURE 3: System structure of cloud-based fault tolerant control.

\section{System Description and Problem Formulation}

2.1. System Model. In order to investigate the active FTC problem for the DC motor system in a cloud environment, a DC motor is considered. The electromechanical dynamics of the DC motor system can be obtained as follows [10]:

$$
\begin{aligned}
& \frac{d i_{\alpha}}{d t}=-\frac{R}{L} i_{\alpha}-\frac{K_{b}}{L} \omega+\frac{1}{L} u_{\alpha}, \\
& \frac{d \omega}{d t}=\frac{K}{J} i_{\alpha}-\frac{B}{J} \omega,
\end{aligned}
$$

where $i_{\alpha}$ is the armature winding current; $\omega$ is the rotor angular speed; $R$ is the armature winding resistance; $L$ is the armature winding inductance; $K_{b}$ is the back electromotive force constant; $u_{\alpha}$ is the armature winding input voltage; $K$ is the torque constant; $J$ is the system moment of inertia; and $B$ is the system damping coefficient.

By introducing a new vector $\mathbf{x}(t)=\left[\begin{array}{ll}i_{\alpha} & \omega\end{array}\right]^{T}$ as the state variable, the DC motor can be rewritten as

$$
\begin{aligned}
& \dot{\mathbf{x}}(t)=\mathbf{A} \mathbf{x}(t)+\mathbf{B u}(t), \\
& \mathbf{y}(t)=\mathbf{C} \mathbf{x}(t)+\mathbf{D} \mathbf{u}(t),
\end{aligned}
$$

where the measurement output vector $\mathbf{y}(t)$ contains the current and rotor angular, and control input signal $\mathbf{u}(t)$ is the input voltage of the system. Furthermore, we have

$$
\mathbf{A}=\left[\begin{array}{rr}
-\frac{R}{L} & -\frac{K_{b}}{L} \\
\frac{K}{J} & -\frac{B}{J}
\end{array}\right],
$$

$$
\begin{aligned}
& \mathbf{B}=\left[\begin{array}{l}
\frac{1}{L} \\
0
\end{array}\right], \\
& \mathbf{C}=\left[\begin{array}{ll}
1 & 0 \\
0 & 1
\end{array}\right], \\
& \mathbf{D}=0 .
\end{aligned}
$$

With a sampling period $h$, the continuous-time model of DC motor can be discretized as

$$
\begin{aligned}
\mathbf{x}(k+1) & =\mathbf{K} \mathbf{x}(k)+\mathbf{G u}(k), \\
\mathbf{y}(k) & =\mathbf{C} \mathbf{x}(k)+\mathbf{D} \mathbf{u}(k),
\end{aligned}
$$

with $\mathbf{K}=e^{\mathbf{A} h}$ and $\mathbf{G}=\int_{0}^{h} e^{\mathbf{A} \tau} d \tau \mathbf{B}$.

2.2. Problem Transformation. From the DC motor model, we have the following discrete-time linear system:

$$
\begin{aligned}
\mathbf{x}(k+1) & =\mathbf{K} \mathbf{x}(k)+\mathbf{G u}(k)+\mathbf{F} \mathbf{f}(k)+\mathbf{S} \boldsymbol{w}(k), \\
\mathbf{y}(k) & =\theta(k) \mathbf{C} \mathbf{x}(k)+\mathbf{E v}(k), \\
\mathbf{z}(k) & =\mathbf{Z} \mathbf{x}(k),
\end{aligned}
$$

where $\mathbf{x}(k) \in \mathbb{R}^{n}$ is the system state; $\mathbf{u}(k) \in \mathbb{R}^{c}$ is the control input vector; $\mathbf{f}(k) \in \mathbb{R}^{f}$ is the fault signal; $\mathbf{y}(k) \in$ $\mathbb{R}^{r}$ represents the measurement output vector, $\boldsymbol{\omega}(k) \in \mathbb{R}^{q}$ and $\mathbf{v}(k) \in \mathbb{R}^{p}$ are uncorrelated zero-mean white external disturbances with $E\left\{\boldsymbol{\omega}(k) \boldsymbol{\omega}(k)^{T}\right\}=\mathbf{W}$ and $E\left\{\mathbf{v}(k) \mathbf{v}(k)^{T}\right\}=\mathbf{V}$, and $\mathbf{z}(k) \in \mathbb{R}^{m}$ is controlled output. The matrices $\mathbf{C}, \mathbf{E}, \mathbf{F}$, 
$\mathbf{S}, \mathbf{K}, \mathbf{G}$, and $\mathbf{Z}$ are real matrices of appropriate dimensions. $\theta(k) \in\{0,1\}$ is a Bernoulli stationary stochastic process with known distribution law at time instant $k$ satisfying

$$
\begin{aligned}
& \operatorname{Pr}\{\theta(k)=1\}=E\{\theta(k)\}=\beta, \\
& \operatorname{Pr}\{\theta(k)=0\}=1-E\{\theta(k)\}=1-\beta,
\end{aligned}
$$

where $\beta$ can be understood as the packet arriving rate, and $1-\beta$ stands for the packet dropout rate.

Remark 1. Compared with the traditional NCSs, the cloudbased control systems are more difficult to handle [31, 32]. This is because the cloud service provider often serves multiple clients simultaneously in the diagnosed cloud process, how to guarantee the exact calculation of residual signal and estimation signal is not easy, and how to guarantee the stability of the whole DC system in a based environment is also hard. In the present paper, the DC system is studied with data missing and actuator fault in a cloud environment.

Before ending this section, we recall the following three assumptions.

Assumption 2. There is at most one faulty actuator at a certain time instant.

Assumption 3. Only additive fault with constant amplitude is considered.

Assumption 4. There are enough computational resource nodes that can be shared in the cloud.

\section{Main Results}

3.1. Redundancy Filter Design. In this section, we propose a redundancy filter design technique that can be used for the fault detection and isolation as well as estimation simultaneously [33].

It follows from Assumption 3 that $\mathbf{f}(k+1)=\mathbf{f}(k)$; we can define

$$
\overline{\mathbf{x}}(k)^{(i)}=\left[\begin{array}{c}
\mathbf{x}(k) \\
\mathbf{T}_{i} \mathbf{f}(k)
\end{array}\right] .
$$

For each, $1 \leq i \leq n_{f}$, we have the overall fault estimation dynamics governed by the following system:

$$
S_{i}:\left\{\begin{array}{l}
\overline{\mathbf{x}}(k+1)^{(i)}=\overline{\boldsymbol{\Gamma}}^{(i)} \overline{\mathbf{x}}(k)^{(i)}+\overline{\mathbf{G}} \mathbf{u}(k)+\overline{\mathbf{S}} \boldsymbol{\omega}(k), \\
\mathbf{y}(k)^{(i)}=\theta(k) \overline{\mathbf{C}} \overline{\mathbf{x}}(k)^{(i)}+\mathbf{E v}(k),
\end{array}\right.
$$

where

$$
\begin{aligned}
\overline{\boldsymbol{\Gamma}}^{(i)} & =\left[\begin{array}{cc}
\mathbf{K} & \mathbf{F T}_{i} \\
0 & \mathbf{T}_{i}
\end{array}\right], \\
\overline{\mathbf{G}} & =\left[\begin{array}{l}
\mathbf{G} \\
0
\end{array}\right],
\end{aligned}
$$

$$
\begin{aligned}
& \overline{\mathbf{S}}=\left[\begin{array}{l}
\mathbf{S} \\
0
\end{array}\right], \\
& \overline{\mathbf{C}}=\left[\begin{array}{ll}
\mathbf{C} & 0
\end{array}\right] .
\end{aligned}
$$

According to (7), a series of matrices $\mathbf{T}_{i}(1 \leq i \leq$ $n_{f}$ ) are employed, and each fault can be described by using $\mathbf{T}_{i} \mathbf{f}(k)\left(1 \leq i \leq n_{f}\right)$ corresponding to one actuator fault, where

$$
\mathbf{T}_{i}:=\operatorname{diag}\{\underbrace{0 \cdots 0}_{i-1}, 1, \underbrace{0 \cdots 0}_{n_{f}-1}\} .
$$

We formally denote $\mathbf{T}_{0}=\mathbf{I}$ and the augmented system $S_{0}$ reflects the evolution rules of augmented states including system state and all possible faults to verify convenient.

For the system (8), consider a series of full-order filter in the following form:

$$
\widehat{\mathbf{x}}(k+1)^{(i)}=\mathbf{K}_{k}^{(i)} \widehat{\mathbf{x}}^{(i)}(k)+\mathbf{L}_{k}^{(i)} \mathbf{y}(k)+\overline{\mathbf{G}} \mathbf{u}(k),
$$

where $i$ stands for the $i$ th fault and $\widehat{\mathbf{x}}(0)^{(i)}=E\left\{\overline{\mathbf{x}}(0)^{(i)}\right\}$. For any $k, \mathbf{K}_{k}^{(i)}$ and $\mathbf{L}_{k}^{(i)}$ are parameters to be determined.

Theorem 5. The filter parameters (11) leading to both the unbiased and least-squares estimations of system (8) are described by

$$
\begin{aligned}
& \mathbf{K}_{k}^{(i)}=\overline{\boldsymbol{\Gamma}}^{(i)}-\beta \mathbf{L}_{k}^{(i)} \overline{\mathbf{C}} \\
& \mathbf{L}_{k}^{(i)}=\mathbf{R}_{k}^{(i)^{T}} \mathbf{Y}_{k}^{(i)^{-1}}
\end{aligned}
$$

where

$$
\begin{aligned}
\mathbf{Y}_{k}^{(i)}= & \beta^{2} \overline{\mathbf{C}} \mathbf{P}_{k}^{(i)} \overline{\mathbf{C}}^{T}+\mathbf{E} \mathbf{V} \mathbf{E}^{T} \\
& +\beta(1-\beta) \overline{\mathbf{C}} \mathbf{\Omega}_{k}^{(i)} \overline{\mathbf{C}}^{T}, \\
\mathbf{R}_{k}^{(i)}= & \beta \overline{\mathbf{C}} \mathbf{P}_{k}^{(i)} \overline{\boldsymbol{\Gamma}}^{(i)^{T}}, \\
\boldsymbol{\alpha}(k+1)^{(i)}= & \overline{\boldsymbol{\Gamma}}^{(i)} \boldsymbol{\alpha}(k)^{(i)}+\overline{\mathbf{G}} \mathbf{u}(k), \\
\boldsymbol{\delta}(k+1)^{(i)}= & \overline{\boldsymbol{\Gamma}}^{(i)} \boldsymbol{\delta}(k)^{(i)} \overline{\boldsymbol{\Gamma}}^{(i)^{T}}+\overline{\mathbf{G}} \mathbf{u}(k) \mathbf{u}(k)^{T} \overline{\mathbf{G}}^{T} \\
& +\overline{\mathbf{S}} \mathbf{W} \overline{\mathbf{S}}^{T}+\overline{\boldsymbol{\Gamma}}^{(i)} \boldsymbol{\alpha}(k)^{(i)} \mathbf{u}(k)^{T} \overline{\mathbf{G}}^{T} \\
& +\overline{\mathbf{G}} \mathbf{u}(k) \boldsymbol{\alpha}(k)^{(i)^{T}} \overline{\boldsymbol{\Gamma}}^{(i)}, \\
\mathbf{P}_{k+1}^{(i)}= & -\mathbf{R}_{k}^{(i)^{T}} \mathbf{Y}_{k}^{(i)^{-1}} \mathbf{R}_{k}^{(i)}+\overline{\boldsymbol{\Gamma}}^{(i)} \mathbf{P}_{k}^{(i)} \overline{\boldsymbol{\Gamma}}^{(i)^{T}}+\overline{\mathbf{S}} \mathbf{W} \overline{\mathbf{S}}^{T} .
\end{aligned}
$$

Proof. Denote $\mathbf{e}(k)^{(i)}=\overline{\mathbf{x}}(k)^{(i)}-\widehat{\mathbf{x}}(k)^{(i)}$. From (8) and (11), one obtains

$$
\begin{aligned}
\mathbf{e}(k+1)^{(i)}= & \overline{\boldsymbol{\Gamma}}^{(i)} \overline{\mathbf{x}}(k)^{(i)}+\overline{\mathbf{S}} \boldsymbol{\omega}(k)-\mathbf{L}_{k}^{(i)} \mathbf{y}(k)^{(i)} \\
& -\mathbf{K}_{k}^{(i)} \widehat{\mathbf{x}}(k)^{(i)}
\end{aligned}
$$


If follows from (12) and $\widehat{\mathbf{x}}(0)^{(i)}=E\left\{\overline{\mathbf{x}}(0)^{(i)}\right\}$ that

$$
\begin{aligned}
E\left\{\mathbf{e}(k+1)^{(i)}\right\}= & \left(\overline{\boldsymbol{\Gamma}}^{(i)}-\beta \mathbf{L}_{k}^{(i)} \overline{\mathbf{C}}\right) E\left\{\mathbf{e}(k)^{(i)}\right\} \\
& -E\{\theta(k)-\beta\} \mathbf{L}_{k}^{(i)} \overline{\mathbf{C}} \overline{\mathbf{x}}(k)^{(i)}=0 ;
\end{aligned}
$$

therefore, the unbiased estimation can be guaranteed.

Denoting $\mathbf{P}_{k}^{(i)}=E\left\{\mathbf{e}(k)^{(i)} \mathbf{e}(k)^{(i)^{T}}\right\}$, it remains to verify that the $\mathbf{L}_{k}^{(i)}$ in (13) minimizes $\mathbf{P}_{k+1}^{(i)}$. Substituting (12) into (19), it is easy to show that

$$
\begin{aligned}
\mathbf{e}(k+1)^{(i)}= & \left(\overline{\boldsymbol{\Gamma}}^{(i)}-\beta \mathbf{L}_{k}^{(i)} \overline{\mathbf{C}}\right) \mathbf{e}(k)^{(i)} \\
& -(\theta(k)-\beta) \mathbf{L}_{k}^{(i)} \overline{\mathbf{C}} \overline{\mathbf{x}}(k)^{(i)}+\overline{\mathbf{S}} \boldsymbol{\omega}(k) \\
& -\mathbf{L}_{k}^{(i)} \mathbf{E v}(k) .
\end{aligned}
$$

Furthermore, we denote $\boldsymbol{\alpha}(k)^{(i)}=E\left\{\overline{\mathbf{x}}(k)^{(i)}\right\}$ and $\boldsymbol{\delta}(k)^{(i)}=$ $E\left\{\overline{\mathbf{x}}(k)^{(i)} \overline{\mathbf{x}}(k)^{(i)^{T}}\right\}$.

From the equation in $(8), \boldsymbol{\alpha}(k)^{(i)}$ and $\boldsymbol{\delta}(k)^{(i)}$ can be recursively updated as (16) and (17) show. Then it follows from (14), (15), and (21) that

$$
\begin{aligned}
\mathbf{P}_{k+1}^{(i)}= & \mathbf{L}_{k}^{(i)} \mathbf{Y}_{k}^{(i)} \mathbf{L}_{k}^{(i)^{T}}-\mathbf{L}_{k}^{(i)} \mathbf{R}_{k}^{(i)}-\mathbf{R}_{k}^{(i)^{T}} \mathbf{L}_{k}^{(i)^{T}} \\
& +\overline{\boldsymbol{\Gamma}}^{(i)} \mathbf{P}_{k}^{(i)} \overline{\boldsymbol{\Gamma}}^{(i)^{T}}+\overline{\mathbf{S}} \mathbf{W} \overline{\mathbf{S}}^{T}
\end{aligned}
$$

Noting that $\mathbf{Y}_{k}^{(i)}=\mathbf{Y}_{k}^{(i)^{T}}>0, \mathbf{P}_{k+1}^{(i)}$ can be expressed as

$$
\begin{aligned}
\mathbf{P}_{k+1}^{(i)}= & \left(\mathbf{L}_{k}^{(i)}-\mathbf{R}_{k}^{(i)^{T}} \mathbf{Y}_{k}^{(i)^{-1}}\right) \mathbf{Y}_{k}^{(i)}\left(\mathbf{L}_{k}^{(i)}-\mathbf{R}_{k}^{(i)^{T}} \mathbf{Y}_{k}^{(i)^{-1}}\right)^{T} \\
& -\mathbf{R}_{k}^{(i)^{T}} \mathbf{Y}_{k}^{(i)^{-1}} \mathbf{R}_{k}^{(i)}+\overline{\boldsymbol{\Gamma}}^{(i)} \mathbf{P}_{k}^{(i)} \overline{\boldsymbol{\Gamma}}^{(i)^{T}}+\overline{\mathbf{S}} \mathbf{W} \overline{\mathbf{S}}^{T} .
\end{aligned}
$$

It is obvious that when $\mathbf{L}_{k}^{(i)}=\mathbf{R}_{k}^{(i)^{T}} \mathbf{Y}_{k}^{(i)^{-1}}$ as in (13), $\mathbf{P}_{k+1}^{(i)}$ is minimized, and the proof of this theorem is now completed.

From Theorem 5, we can obtain a solution for fault estimation for the DC motor system in a cloud-based control system with packet dropout and actuator fault. If the filter design result can be obtained accurately, the fault detection and fault isolation problems will be obtained.

3.2. Fault Detection, Isolation, and Estimation. Theorem 5 offers a solution of the fault detection and isolation as well as estimation in a unified way. A series of filters is designed to estimate the augmented signal composed of the system state and a specific fault signal, where each filter is implemented by a certain computational node in the cloud. It can be inferred from Theorem 5 that each filter is proposed in a recursive way so the whole technique is easy to be carried out. The main idea for the fault detection, isolation, and estimation is that the filter with the least residual signal is regarded as corresponding to the right fault signal, and its estimation is chosen as the occurred fault.
For system (5), the main idea for fulfilling the fault detection and isolation task from Theorem 5 is to design a series of filters in the cloud with residual signals $J_{k}^{(i)}:=\| \mathbf{y}(k)-$ $\overline{\mathbf{C}}(k) \widehat{\mathbf{x}}(k)^{(i)} \|^{2}$. We employ $J_{k}^{(0)}$ for the fault detection and the rest of the signals can be used for fault isolation by a certain computational node in the cloud.

By choosing a proper threshold $J_{\text {th }}^{0}$, the fault detection can be carried out from the following logic:

$$
\begin{aligned}
& J_{k}^{(0)} \leq J_{\text {th }}^{0}, \quad \text { no fault, } \\
& J_{k}^{(0)} \geq J_{\text {th }}^{0}, \quad \text { fault detected, }
\end{aligned}
$$

where the threshold $J_{k}^{(0)}$ can be determined by the literature $[34,35]$.

The redundant filtering strategy is proposed for the fault isolation problem. Among all of the filters $F_{i}$ we consider the filter with the least filtering error as the one corresponding to the fault correctly, and therefore the fault isolation can be achieved from the following logic:

$$
i=\arg \min _{1 \leq i \leq n_{f}} J_{k}^{(i)}
$$

Apparently, when the fault isolation is achieved, a part of the output of the normal filter can be regarded to be the fault estimation from the augmented system state.

Remark 6. The fault estimation signal of the fault estimation filters with least filtering error generates the real fault. The fault isolation strategy is based on the correct fault estimation, which is referred to as residual matching. Based on a series of fault estimation filters, we can obtain the right fault signal; therefore, the fault can be further located. Furthermore, the estimation of the fault can also be determined.

Once the fault detection, isolation, and estimation are achieved, we can further consider the fault tolerant control in the next subsection.

3.3. Fault Accommodation. The normal controller is given as

$$
\mathbf{u}_{h}(k)=\mathbf{H y}(k),
$$

where $\mathbf{H}$ is the control gain.

Assumption 7. $\operatorname{rank}(\mathbf{G}, \mathbf{F})=\operatorname{rank}(\mathbf{G})$.

Remark 8. According to Assumption 4, it can be easily seen that the vector space spanned by the columns of $\mathbf{F}$ is a subset of the space spanned by the column vectors of $\mathbf{G}$, such as $\operatorname{span}(\mathbf{F}) \subseteq \operatorname{span}(\mathbf{G})$, which is equivalent to the existence of $\mathbf{G}^{*}$ such that $\left(\mathbf{I}-\mathbf{G G}^{*}\right) \mathbf{F}=0$.

Once a fault occurs, based on the accurate and rapid estimation of the fault, the following fault tolerant controller is activated to compensate for the fault:

$$
\mathbf{u}(k)=\mathbf{u}_{h}(k)-\mathbf{G}^{*} \mathbf{F} \widehat{\mathbf{f}}(k),
$$


where $\widehat{\mathbf{f}}(k)$ is the estimation of actuator bias fault. Further we have the following closed-loop system of (5) and (27):

$$
\begin{aligned}
\mathbf{x}(k+1) & =\widetilde{\boldsymbol{\Gamma}} \mathbf{x}(k)+(\theta(k)-\beta) \boldsymbol{\Gamma}_{1} \mathbf{x}(k)+\widetilde{\mathbf{S}} \mathbf{w}_{1}(k), \\
\mathbf{z}(k) & =\mathbf{Z} \mathbf{x}(k),
\end{aligned}
$$

where $\widetilde{\mathbf{\Gamma}}=\mathbf{K}+\beta \mathbf{G H C}, \boldsymbol{\Gamma}_{1}=\mathbf{G H C}$, and $\widetilde{\mathbf{S}}=[\mathbf{G H E}, \mathbf{S},-\mathbf{F}]$.

$$
\boldsymbol{\omega}_{1}(k)=\left[\mathbf{v}(k)^{T}, \boldsymbol{\omega}(k)^{T}, \widehat{\mathbf{f}}(k)^{T}-\mathbf{f}(k)^{T}\right]^{T} .
$$

We consider a controller that can minimize the $\mathbf{H}_{\infty}$ attenuation level from the disturbance $\boldsymbol{\omega}_{1}(k)$ to $\mathbf{z}(k)$. Once the actuator failure is determined, the control law is switched to (27). We aim to guarantee the closed-loop system (5) asymptotically mean square stability when the actuator failure happens and the following performance is satisfied:

$$
\sum_{k=0}^{\infty} E\left\{\|\mathbf{z}(k)\|^{2}\right\} \leq \gamma_{1}^{2} \sum_{k=0}^{\infty} E\left\{\left\|\boldsymbol{\omega}_{1}(k)\right\|^{2}\right\} .
$$

Theorem 9. Consider system (5) with stochastic packet dropout and the actuator fault. There exists a control law (27) such that the closed-loop system described by (5) and (27) is asymptotically mean square stable and the prescribed $\mathbf{H}_{\infty}$ disturbance attenuation level (30) is fulfilled if there exist matrices $\mathbf{X}=\mathbf{X}^{T}>0, \overline{\mathbf{H}} \in \mathbb{R}^{n_{x}}$, and a scalar $\gamma_{1}>0$ such that the following LMI holds:

$$
\left[\begin{array}{ccccccc}
-\mathbf{X} & 0 & \sqrt{(1-\beta) \beta} \mathbf{G} \overline{\mathbf{H}} & 0 & 0 & 0 & 0 \\
* & -\mathbf{X} & \mathbf{\Gamma X}+\beta \mathbf{G} \overline{\mathbf{H}} & \mathbf{G H E} & \mathbf{S} & \mathbf{F} & 0 \\
* & * & -\mathbf{X} & 0 & 0 & 0 & \mathbf{X Z}^{T} \\
* & * & * & -\gamma_{1}^{2} \mathbf{I} & 0 & 0 & 0 \\
* & * & * & * & -\gamma_{1}^{2} \mathbf{I} & 0 & 0 \\
* & * & * & * & * & -\gamma_{1}^{2} \mathbf{I} & 0 \\
* & * & * & * & * & * & -\mathbf{I}
\end{array}\right]
$$

$<0$.

If (31) holds, the desired control law can be given by the following equation:

$$
\mathbf{H}=\overline{\mathbf{H}} \mathbf{X}^{-1} \mathbf{C}^{-1},
$$

where $\mathbf{C}^{-1}$ is the left inverse matrix of $\mathbf{C}$.

Proof. Consider the following Lyapunov function $V_{k}=$ $x_{k}^{T} P x_{k}$ and an $\mathbf{H}_{\infty}$ attenuation level. For any initial condition and initial distribution, $\omega_{1}(k) \neq 0$, we can obtain

$$
\begin{aligned}
\Delta V(k)= & E\left\{x(k+1)^{T} P x(k+1)\right\}-x(k)^{T} P x(k) \\
& +E\left\{z(k+1)^{T} z(k+1)\right\} \\
& -\gamma_{1}^{2} E\left\{\omega_{1}(k)^{T} \omega_{1}(k)\right\}
\end{aligned}
$$

$$
\begin{aligned}
= & x(k)^{T}\left[\widetilde{\Gamma}^{T} P \widetilde{\Gamma}+(1-\beta) \beta \Gamma_{1}{ }^{T} P \Gamma_{1}-P\right] x(k) \\
& -\gamma_{1}^{2} \omega_{1}(k)^{T} \omega_{1}(k)+\widetilde{S}^{T} \widetilde{S} \omega_{1}(k)^{T} \omega_{1}(k) \\
& +x(k)^{T} Z^{T} Z x(k) .
\end{aligned}
$$

The right side of equation (33) is equivalent to (34) by the Schur complement.

$$
\left[\begin{array}{ccccc}
-P & 0 & \sqrt{(1-\beta) \beta} P \Gamma_{1} & 0 & 0 \\
* & -P & -P \widetilde{\Gamma} & -P \widetilde{S} & 0 \\
* & * & -P & 0 & Z^{T} \\
* & * & * & -\gamma_{1}^{2} I & 0 \\
* & * & * & * & -I
\end{array}\right]<0 .
$$

Let $X=P^{-1}$; we premultiply and postmultiply (34) by $\operatorname{diag}\{X, X, X, I, I, I, I\}$ and its transpose, and after some proper elementary transformation, we can get

$$
\left[\begin{array}{ccccccc}
-X & 0 & \sqrt{(1-\beta) \beta} G H C X & 0 & 0 & 0 & 0 \\
* & -X & \Gamma X+\beta G H C X & G H E & S & F & 0 \\
* & * & -X & 0 & 0 & 0 & X Z^{T} \\
* & * & * & -\gamma_{1}^{2} I & 0 & 0 & 0 \\
* & * & * & * & -\gamma_{1}^{2} I & 0 & 0 \\
* & * & * & * & * & -\gamma_{1}^{2} I & 0 \\
* & * & * & * & * & * & -I
\end{array}\right]
$$

Considering $\bar{H}=H C X$ and from Theorem 9, we can infer (35) holds, which indicates $\Delta V(k)<0$ in (33). We further ensure that system (28) is mean square stable and satisfies the constraint (30). If (31) has solutions and $C, X$ are nonsingular, the control gain can be obtained by (32). This concludes the proof.

Based on the previous discussions, the whole FTC problem for the discrete-time linear system in a cloud environment with packet dropout and actuator fault can be dealt with by using the following algorithm.

\section{Algorithm 10.}

Step 1. Determine the parameters of a fault estimator (11) by using Theorem 5.

Step 2. Design a series of least-square fault detection filters $F_{i}\left(0 \leq i \leq n_{f}\right)$. The filter $F_{0}$ generates a fault estimation signal, which can be used to indicate the fault.

Step 3. Choose the filter $F_{i}\left(1 \leq i \leq n_{f}\right)$ with the smallest filtering error signal by comparing with the filter $F_{i}$ and determine the fault location $i$ by a certain computational 
TABle 1: Parameters of the DC motor [10].

\begin{tabular}{lcc}
\hline$J$ & Inertia & $1.6511 \times 10^{-1} \mathrm{~kg} / \mathrm{m}^{2}$ \\
$L$ & Inductance & $3.14 \times 10^{-3} \mathrm{H}$ \\
$R$ & Resistance & $4.961 \Omega$ \\
$K$ & Torque constant & $7.105 \times 10^{-2} \mathrm{~N} \cdot \mathrm{m} / \mathrm{A}$ \\
$B$ & Damping coefficient & $2.364 \times e^{-5} \mathrm{~N} \cdot \mathrm{m} \cdot \mathrm{sec} / \mathrm{rad}$ \\
$K_{b}$ & Back-EMF constant & $1.276 \times 10^{-2} \mathrm{Vs} / \mathrm{rad}$ \\
\hline
\end{tabular}

node in the cloud. Take the output of the filter $F_{i}$ as the fault estimation result.

Step 4. For the fault-free case and the faulty case with fault estimation information, utilize Theorem 9 to calculate the control gain $\mathbf{H}$.

Step 5. Utilize the estimated fault information to adjust the control law designed in Step 4.

\section{Simulation Results}

To illustrate the effectiveness of the proposed FTC method, experimental simulations are conducted based on the DC motor system reported in [10]. In this paper, two additive faults each of which affects a specific state are considered. The problem in the present study is to design the fault diagnosis unit and carry out an active fault tolerant control in the presence of packet dropout and actuator fault in the DC motor system in a cloud environment. From Assumption 3, when the fault happens, the fault system is constructed as

$$
\begin{aligned}
\mathbf{x}(k+1) & =\mathbf{K} \mathbf{x}(k)+\mathbf{G u}(k)+\sum_{s=1}^{n_{f}} b_{s} \mathbf{f}_{s}(k)+\mathbf{S} \boldsymbol{\omega}(k), \\
\mathbf{y}(k) & =\theta(k) \mathbf{C x}(k)+\mathbf{E v}(k) \\
\mathbf{z}(k) & =\mathbf{Z} \mathbf{x}(k)
\end{aligned}
$$

where $b_{s}(s=1,2)$ is the $s$ th column of matrix $\mathbf{F}$. The parameters of the DC motor used in this paper are given in Table 1. The sampling period of the DC motor system is specified to $0.1 \mathrm{~s}$. We set the receiving rate $\theta(k)=0.8$ and choose the system noise and measurement noise with second moments $\mathbf{W}=10^{-6}, \mathbf{V}=10^{-2}$ in the simulation.

After linearization process, we can get the following parameters of the discrete-time model (5):

$$
\begin{aligned}
& \mathbf{K}=\left[\begin{array}{cc}
-0.0017 & -0.0016 \\
0.6532 & 0.6169
\end{array}\right], \\
& \mathbf{G}=\mathbf{S}=\left[\begin{array}{c}
0.1336 \\
26.5699
\end{array}\right], \\
& \mathbf{F}=\left[\begin{array}{cc}
0.1336 & 0 \\
0 & 26.5699
\end{array}\right],
\end{aligned}
$$

$$
\begin{aligned}
& \mathbf{C}=\mathbf{E}=\left[\begin{array}{ll}
1 & 0 \\
0 & 1
\end{array}\right], \\
& \mathbf{Z}=\left[\begin{array}{l}
0.6 \\
0.4
\end{array}\right] .
\end{aligned}
$$

Further, we have the following vectors in (36):

$$
\begin{aligned}
& b_{1}=\left[\begin{array}{c}
0.1336 \\
0
\end{array}\right], \\
& b_{2}=\left[\begin{array}{c}
0 \\
26.5699
\end{array}\right] .
\end{aligned}
$$

By using Theorem 9, we can obtain the control gain $H=[-0.0273,-0.0258]$. Consider time instant $0 \leq k \leq$ 300. The two-dimensional actuator fault signal $\mathbf{f}(k)=$ $\left[\mathbf{f}_{1}(k)^{T} \mathbf{f}_{2}(k)^{T}\right]^{T}$ is set to be

$$
\begin{aligned}
& \mathbf{f}_{1}(k)=0, \\
& \mathbf{f}_{2}(k)= \begin{cases}0, & k \leq 50, \\
0.01, & \text { otherwise. }\end{cases}
\end{aligned}
$$

That is, the second actuator suffers an additive bias fault from the time instant $k=51$. By using Theorem 5, we design three recursive filters $F_{0}, F_{1}$, and $F_{2}$. Based on the filter bank, we shall conduct simulation results on the fault detection and fault isolation, as well as fault estimation, for the actuator fault of the DC motor system. In the simulation, Figure 4 is the incremental residual signal of $J_{k}^{0}$ and the threshold is obtained as $J_{\text {th }}^{0}=0.0927$ after 200 Monte Carlo simulations. The filters $F_{1}, F_{2}$ are used to isolate the fault. In Figure 5, the residual of filter $F_{2}$ is the least one, and we can confirm actuator 2 is faulty. The fault estimation is shown in Figure 6.

Figure 7 indicates the controlled outputs in two cases. The blue line shows that the DC motor system uses control law $\mathbf{H}$ and there is neither fault diagnosis unit nor fault tolerant strategy. The red line shows that the system uses the fault tolerant control technique proposed in the paper. It can be observed from the simulation results that the system using the fault tolerant control can achieve a better performance than the control target after the occurrence of the fault in actuator 2 without fault accommodation ability.

\section{Conclusion}

In this paper, a fault diagnosis unit and FTC strategy have been designed for a DC motor in a cloud environment. A residual signal from the residual generator has been established for the fault detection and isolation in the presence of packet dropout and actuator fault. A fault estimator has been proposed to provide the joint estimation of system state and the fault signal. Once the actuator fault is confirmed, its estimation information can be obtained from the corresponding filter in least-squares sense, and this information 


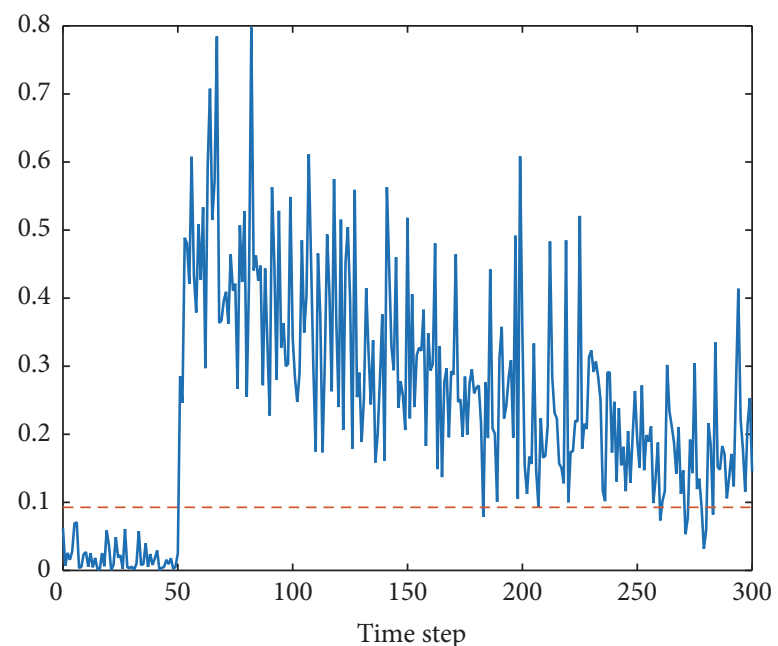

- Residual 0

FIGURE 4: Fault detection.

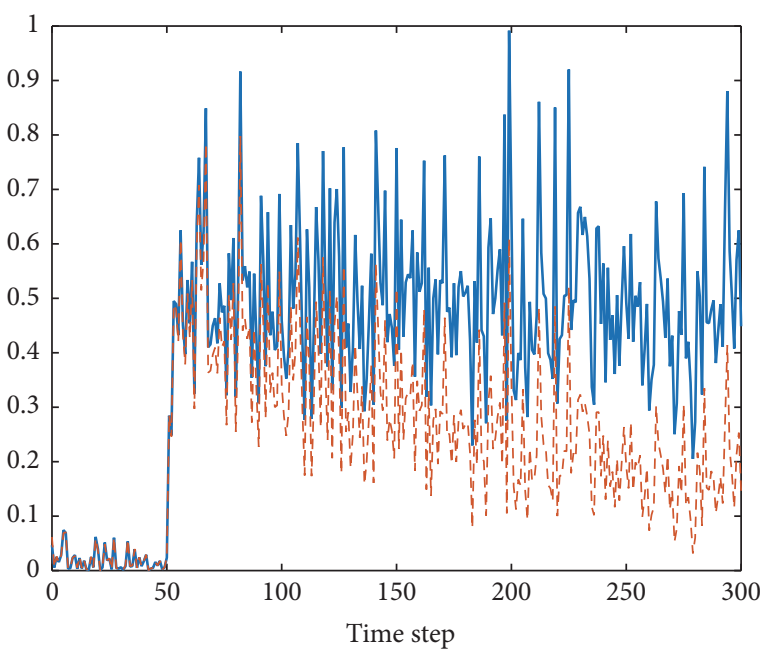

$\begin{array}{cc}\text { - Residual } 1 \\ \text {-- } & \text { Residual } 2\end{array}$

Figure 5: Fault isolation.

can further be adopted to realize a reconfigurable control law. From the simulation results, we can observe that the FTC strategy shows better performance for the DC motor system with the existence of packet dropout and actuator fault in the cloud environment. In future work, we will consider some promising topics including (1) fault estimation and accommodation for nonlinear systems in the cloud environment; (2) enhancing the robustness and reliability of the fault diagnosis and fault tolerant control technologies in the cloud environment; and (3) theoretical analysis of the relationship between determined thresholds and the fault detection and the fault isolation performance.

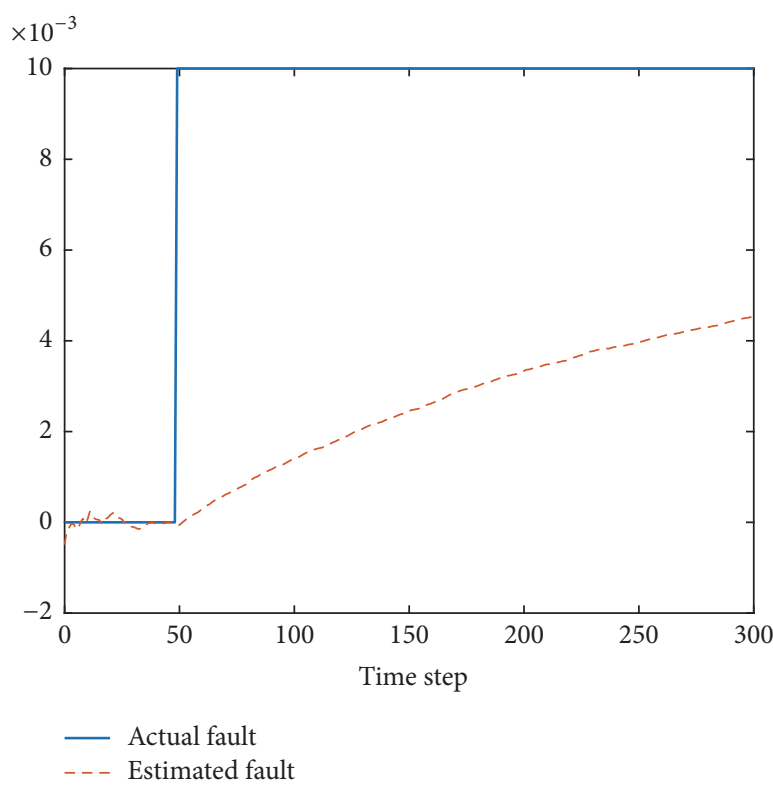

Figure 6: Fault estimation.

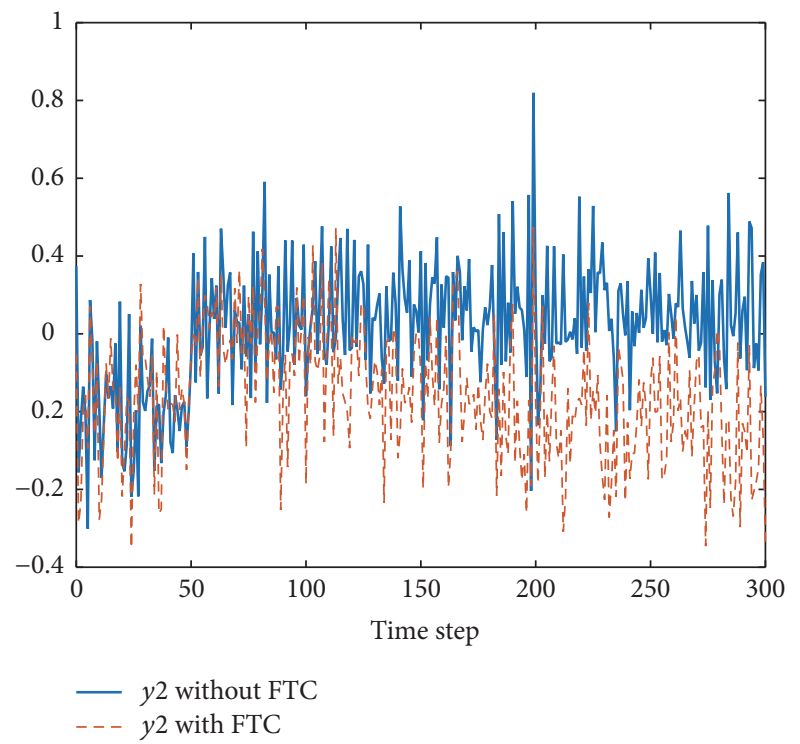

FIGURE 7: Controlled output.

\section{Acknowledgments}

This work was supported by the National Natural Science Foundation of China under Grants 61473163 and 61522309 and open funding from Guangdong Provincial Key Laboratory of Petrochemical Equipment and Fault Diagnosis under Grant GDUPTKLAB201503. The work was also supported by Science and Technology Development Program of Jilin Province under Grant 20150203017G X and Provincial Economic Structure Strategic Adjustment Guidance Fund of Jilin Province under Grant 2015Y063. The work of Yang Liu was supported by China Postdoctoral Science Foundation under Grant 2016M600546 and Qingdao Postdoctoral Applied Research Projects under Grant 2016112. 


\section{Conflicts of Interest}

The authors declare that they have no conflicts of interest.

\section{References}

[1] K. C. Lee, S. Lee, and M. H. Lee, "QoS-based remote control of networked control systems via profibus token passing protocol," IEEE Transactions on Industrial Informatics, vol. 1, no. 3, pp. 183191, 2005.

[2] D. Yue, E. Tian, and Q.-L. Han, "A delay system method for designing event-triggered controllers of networked control systems," IEEE Transactions on Automatic Control, vol. 58, no. 2, pp. 475-481, 2013.

[3] W. Zhang, M. S. Branicky, and S. M. Phillips, "Stability of networked control systems," IEEE Control Systems Magazine, vol. 21, no. 1, pp. 84-99, 2001.

[4] Y. Xia, "Cloud control systems," IEEE/CAA Journal of Automatica Sinica, vol. 2, no. 2, pp. 134-142, 2015.

[5] Y. Xia, Y. Qin, D.-H. Zhai, and S. Chai, "Further results on cloud control systems," Science China Information Sciences, vol. 59, no. 7, Article ID 073201, 2016.

[6] H. R. Karimi, "Robust synchronization and fault detection of uncertain master-slave systems with mixed time-varying delays and nonlinear perturbations," International Journal of Control, Automation and Systems, vol. 9, no. 4, pp. 671-680, 2011.

[7] G.-P. Liu, Y. Xia, J. Chen, D. Rees, and W. Hu, "Networked predictive control of systems with random network delays in both forward and feedback channels," IEEE Transactions on Industrial Electronics, vol. 54, no. 3, pp. 1282-1297, 2007.

[8] P. Seiler and R. Sengupta, "An $H_{\infty}$ approach to networked control," IEEE Transactions on Automatic Control, vol. 50, no. 3, pp. 356-364, 2005.

[9] C. Lazar and S. Carari, "A remote-control engineering laboratory," IEEE Transactions on Industrial Electronics, vol. 55, no. 6, pp. 2368-2375, 2008.

[10] H. Li, M.-Y. Chow, and Z. Sun, "EDA-based speed control of a networked DC motor system with time delays and packet losses," IEEE Transactions on Industrial Electronics, vol. 56, no. 5, pp. 1727-1735, 2009.

[11] Z. Du, D. Yue, and S. Hu, "H- $\mathrm{H}$ stabilization for singular networked cascade control systems with state delay and disturbance," IEEE Transactions on Industrial Informatics, vol. 10, no. 2, pp. 882-894, 2014.

[12] Y. Luo, G. Wei, Y. Liu, and X. Ding, "Reliable $H_{\infty}$ state estimation for 2-D discrete systems with infinite distributed delays and incomplete observations," International Journal of General Systems, vol. 44, no. 2, pp. 155-168, 2015.

[13] M. Blanke, M. Kinnaert, J. Lunze, and M. Staroswiecki, Diagnosis and Fault-Tolerant Control, Springer, Berlin, Germany, 2006.

[14] F. Pierri, G. Paviglianiti, F. Caccavale, and M. Mattei, "Observerbased sensor fault detection and isolation for chemical batch reactors," Engineering Applications of Artificial Intelligence, vol. 21, no. 8, pp. 1204-1216, 2008.

[15] X. Wei, M. Verhaegen, and T. van Engelen, "Sensor fault detection and isolation for wind turbines based on subspace identification and Kalman filter techniques," International Journal of Adaptive Control and Signal Processing, vol. 24, no. 8, pp. 687-707, 2010.

[16] A. Ray, M. Desai, and J. Deyst, "Fault detection and isolation in a nuclear reactor," Journal of Energy, vol. 7, no. 1, pp. 79-85, 1983.
[17] C. Edwards, S. K. Spurgeon, and R. J. Patton, "Sliding mode observers for fault detection and isolation," Automatica, vol. 36, no. 4, pp. 541-553, 2000.

[18] A. Q. Khan and S. X. Ding, "Threshold computation for fault detection in a class of discrete-time nonlinear systems," International Journal of Adaptive Control and Signal Processing, vol. 25, no. 5, pp. 407-429, 2011.

[19] Y. Song, J. Hu, D. Chen, D. Ji, and F. Liu, "Recursive approach to networked fault estimation with packet dropouts and randomly occurring uncertainties," Neurocomputing, vol. 214, pp. 340349, 2016.

[20] A. Farhat and D. Koenig, "PI robust fault detection observer for a class of uncertain switched systems using LMIs," International Federation of Automatic Control-Papers On Line, vol. 48, no. 21, pp. 125-130, 2015.

[21] R. Marino, S. Scalzi, P. Tomei, and C. M. Verrelli, "Fault-tolerant cruise control of electric vehicles with induction motors," Control Engineering Practice, vol. 21, no. 6, pp. 860-869, 2013.

[22] C. Bonivento, A. Isidori, L. Marconi, and A. Paoli, "Implicit fault-tolerant control: application to induction motors," Automatica, vol. 40, no. 3, pp. 355-371, 2004.

[23] S. X. Ding, P. Zhang, S. Yin, and E. L. Ding, "An integrated design framework of fault-tolerant wireless networked control systems for industrial automatic control applications," IEEE Transactions on Industrial Informatics, vol. 9, no. 1, pp. 462-471, 2013.

[24] J. You, S. Yin, and H. Gao, "Fault detection for discrete systems with network-induced nonlinearities," IEEE Transactions on Industrial Informatics, vol. 10, no. 4, pp. 2216-2223, 2014.

[25] B. Jiang and F. N. Chowdhury, "Parameter fault detection and estimation of a class of nonlinear systems using observers," Journal of the Franklin Institute, vol. 342, no. 7, pp. 725-736, 2005.

[26] H. Yang, Y. Xia, and P. Shi, "Observer-based sliding mode control for a class of discrete systems via delta operator approach," Journal of the Franklin Institute, vol. 347, no. 7, pp. 1199-1213, 2010.

[27] J. Blesa, D. Rotondo, V. Puig, and F. Nejjari, "FDI and FTC of wind turbines using the interval observer approach and virtual actuators/sensors," Control Engineering Practice, vol. 24, no. 1, pp. 138-155, 2014.

[28] J.-S. Wang and G.-H. Yang, "Data-driven output-feedback faulttolerant control for unknown dynamic systems with faults changing system dynamics," Journal of Process Control, vol. 43, pp. 10-23, 2016.

[29] S. Wang, J. Feng, and H. Zhang, "Robust fault tolerant control for a class of networked control systems with state delay and stochastic actuator failures," International Journal of Adaptive Control and Signal Processing, vol. 28, no. 9, pp. 798-811, 2014.

[30] D. Krokavec, A. Filasová, and V. Serbák, "FTC structures with virtual actuators and dynamic output controllers," International Federation of Automatic Control-Papers On Line, vol. 48, no. 21, pp. 511-516, 2015.

[31] J. M. Bradley and E. M. Atkins, "Toward continuous state-space regulation of coupled cyber-physical systems," Proceedings of the IEEE, vol. 100, no. 1, pp. 60-74, 2012.

[32] Y. Q. Xia, "From networked control systems to cloud control systems," in Proceedings of the 31st Chinese Control Conference, pp. 5878-5883, IEEE, Kunming, China, July 2012.

[33] X. He, Z. Wang, Y. Liu, and D. H. Zhou, "Least-squares fault detection and diagnosis for networked sensing systems 
using a direct state estimation approach," IEEE Transactions on Industrial Informatics, vol. 9, no. 3, pp. 1670-1679, 2013.

[34] X. He, Z. Wang, and D. H. Zhou, "Robust fault detection for networked systems with communication delay and data missing," Automatica, vol. 45, no. 11, pp. 2634-2639, 2009.

[35] X. He, Z. Wang, Y. Liu, L. Qin, and D. Zhou, "Fault tolerant control for an internet-based three-tank system: accommodation to sensor bias faults," IEEE Transactions on Industrial Electronics, vol. PP, no. 99, pp. 1-1, 2016. 


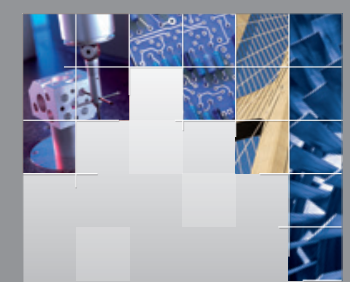

\section{Enfincering}
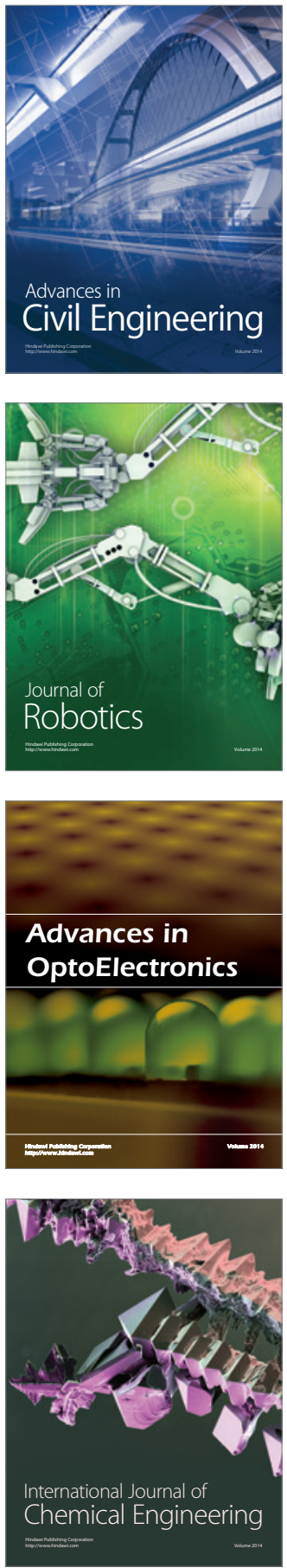

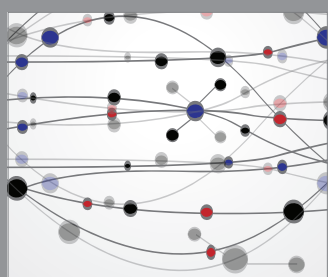

The Scientific World Journal

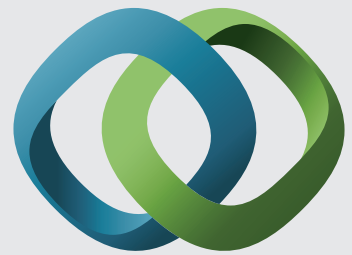

\section{Hindawi}

Submit your manuscripts at

https://www.hindawi.com
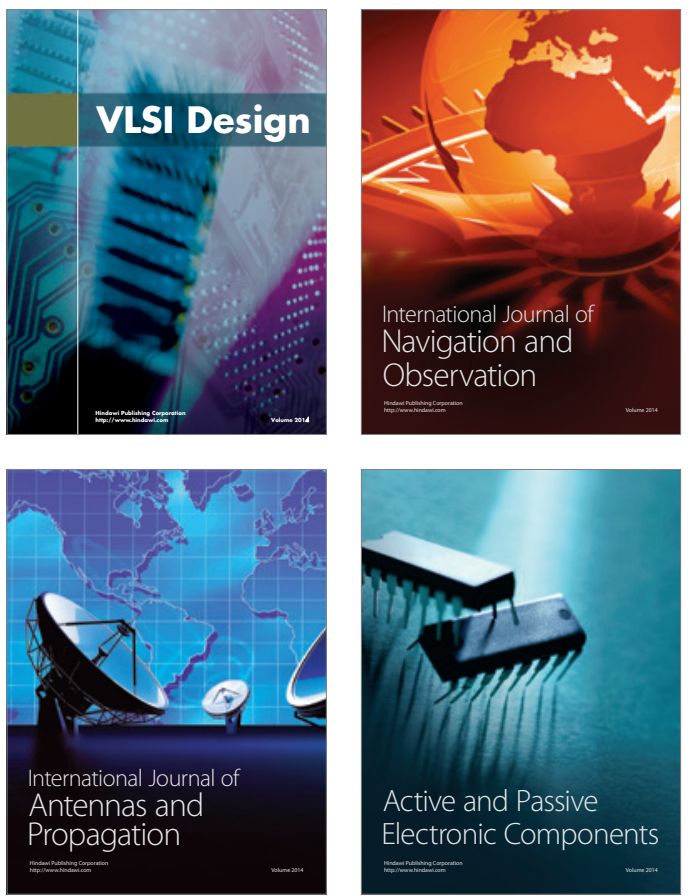
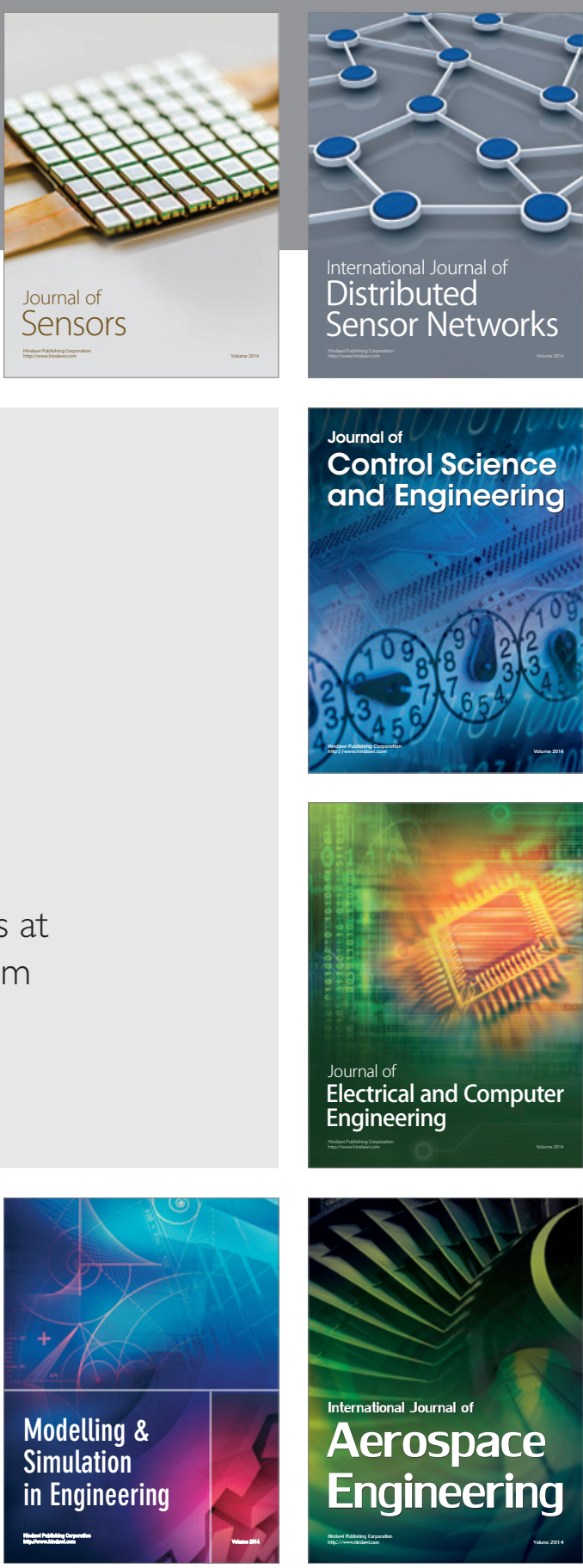

International Journal of

Distributed

Sensor Networks

$-$

Joumal of

Control Science

and Engineering
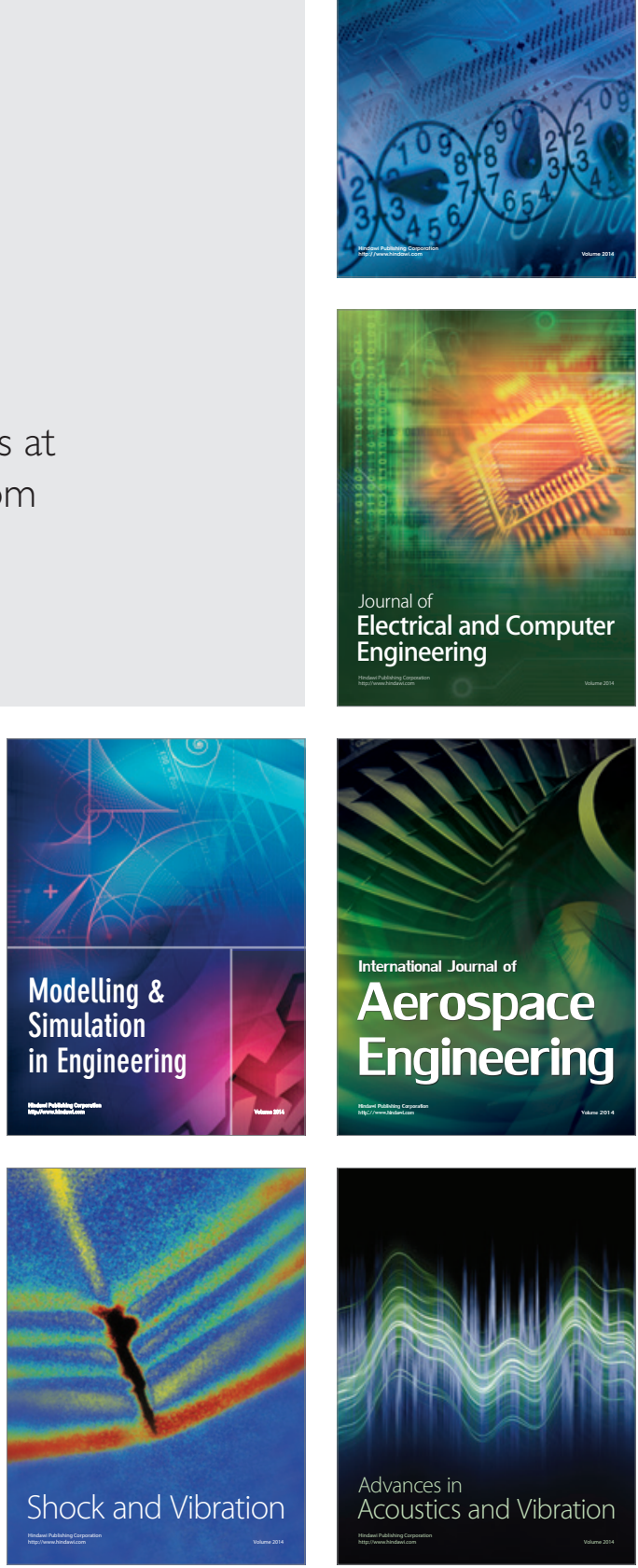